\title{
Phase behavior of colloid plus polydisperse polymer mixtures
}

\author{
Richard P. Sear* and Daan Frenkel ${ }^{\dagger}$ \\ FOM Institute for Atomic and Molecular Physics, Kruislaan 407, NL-1098 SJ Amsterdam, The Netherlands
}

(Received 26 June 1996)

\begin{abstract}
A suspension of colloidal particles and polydisperse polymer coils is modeled using the hard-sphere potential for the colloid and the Asakura-Oosawa model for the colloid-polymer interaction [S. Asakura and F. Oosawa, J. Chem. Phys. 22, 1255 (1954)]. The phase behavior of the mixtures is compared with that for a monodisperse polymer [H. N. W. Lekkerkerker et al., Europhys. Lett. 20, 559 (1992)]. Polydispersity is seen to increase the extent of the fluid-fluid coexistence found if the radius of gyration of the polymer is sufficiently large. The partitioning of polymer coils of different sizes between coexisting phases is studied. [S1063-651X(97)08701-1]

PACS number(s): 82.70.Dd, 64.60.-i
\end{abstract}

\section{INTRODUCTION}

Man-made polymers are inevitably polydisperse. However, once the polydispersity is reduced as much as possible the polymer is often treated as if it were completely monodisperse. This is done because polydisperse systems are much more complex than their monodisperse counterparts and much less well defined. The theoretical calculation of first-order phase transitions in polydisperse systems is particularly difficult due to the partitioning of the different polymer species among the coexisting phases [1]. For example, at the isotropic-nematic transition of a liquid-crystalline polymer the polymer molecules of different lengths will be partitioned between the isotropic and nematic phases in such a way as to minimize the total free energy, subject to conservation of the total number of polymer molecules of each length in the system. So, at constant pressure, while we need to solve only one nonlinear equation for a monodisperse system, we need to perform a functional minimization for the polydisperse equivalent. In experiment the partitioning of the polymer occurs spontaneously, but there remains the problem of the polydisperse system being less well defined and understood than a pure component or even a binary mixture.

A natural question to ask is: Is the complexity of polydisperse systems hiding some interesting and perhaps even useful behavior? Here we address this question for a specific mixture: a mixture of colloidal particles and polydisperse polymer [2]. We find some, perhaps unexpected, results such as a more pronounced phase separation in polydisperse systems than in an "equivalent" monodisperse system. Thus, if the polymer is added to the colloidal suspension specifically to induce phase separation, it may be advantageous to use a polydisperse polymer in place of a monodisperse one. Conversely, when polymer is used in protein crystallization, it is believed to be important [3] to avoid phase separation. Then, it may be helpful to use a polymer that is as monodisperse as possible. Equivalent is in quotations because no polydisperse system is truly equivalent to any monodisperse system; for any polydisperse system there is no unique monodisperse

\footnotetext{
*Electronic address: sear@amocf6.amolf.nl

†Electronic address: frenkel@amocf3.amolf.nl
}

system with that it may be compared. However, we can compare a polydisperse system to one which is monodisperse but has the same average value of a quantity such as molecular weight.

In the following section we generalize the theory of Lekkerkerker et al. [4] to include a polydisperse polymer. Section III contains some example phase diagrams along with a discussion and Sec. IV stresses the potential usefulness of polydispersity.

\section{MODEL AND THEORY}

Our models for the interaction between colloidal particles and between colloidal particles and polymers are standard. The colloidal particles are spherical and interact with each other as hard spheres of diameter $\sigma[2,5]$; see Fig. 1(b). The colloid is assumed to be monodisperse. The colloid-polymer interaction is also modeled as a hard-sphere interaction: the Asakura-Oosawa model [6-8]. Each colloidal sphere excludes the center of a polymer coil of size $\varepsilon$ from a sphere of diameter $\sigma(1+\varepsilon)$ centered on the colloidal sphere; see Fig. $1(\mathrm{a}) . \varepsilon$ is roughly equal to twice the radius of gyration of a polymer molecule [8,9], in units of $\sigma$. The polymer is assumed ideal, i.e., polymer-polymer interactions are ignored.

A polydisperse mixture is a many-component mixture in the limit that the number of components tends to infinity (see Refs. $[1,10,11])$ for the formalism of polydisperse mixtures. In the canonical ensemble our mixture is specified by the temperature $T$, volume $V$, number of colloidal spheres $N_{c}$, and density function of the polymer $\rho_{p}(\varepsilon)$. The density func-

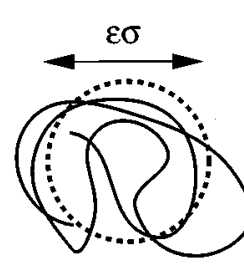

(a)

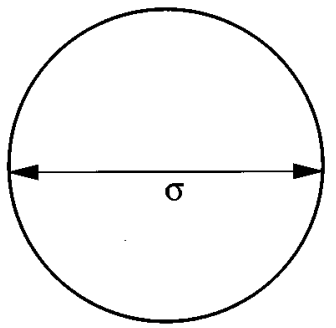

(b)
FIG. 1. Models for the (a) polymer and (b) colloid. The dashed circle in (a) represents the effective hard-sphere diameter for the colloid-polymer interaction. 
tion $\rho_{p}(\varepsilon)$ is defined such that the number density of polymer coils of size $\varepsilon$ is $\rho_{p}(\varepsilon) d \varepsilon$. The chemical potential of the polymer is given by an activity function $z_{p}(\varepsilon)$, where $z_{p}(\varepsilon)$ is the activity of polymer coils of size $\varepsilon$. Finding phase coexistence in a polydisperse mixture using the canonical ensemble is complicated by the fact that it is necessary to vary the functions $\rho_{p}(\varepsilon)$ in the coexisting phases in order that the functions $z_{p}(\varepsilon)$ be the same in each phase $[1,10]$. Here, however, because the polymer coils do not interact with each other it is a simple matter to transform from the canonical ensemble to a semigrand ensemble $[4,9,12]$ in which the polymer is specified not by $\rho_{p}(\varepsilon)$ but by $z_{p}(\varepsilon)$. The variables are then $N_{c}, V, T$, and $z_{p}(\varepsilon)$ and determining phase coexistence is no more difficult than in a pure fluid; all that is necessary is to equate the pressure and the chemical potential of the colloid.

The semigrand potential $\Omega$ is given by a straightforward generalization of that used for monodisperse polymer (see Refs. $[4,8,9])$

$$
\beta \Omega\left(N_{c}, V ; z_{p}\right]=\beta A_{c}\left(N_{c}, V\right)-V \int_{0}^{\infty} d(\varepsilon) z_{p}(\varepsilon) \alpha\left(\varepsilon, N_{c} / V\right),
$$

where $\beta=1 / k_{B} T$, with $k_{B}$ Boltzmann's constant, and $A_{c}$ is the Helmholtz free energy of a pure system of colloidal spheres. $\alpha\left(\varepsilon, N_{c} / V\right)$ is the free volume of a polymer with size $\varepsilon$ in a system of colloidal spheres of density $N_{c} / V$. Equation (1) is, of course, approximate [8]. For $A_{c}$ in the fluid phase we use a free energy derived from the CarnahanStarling [13] equation for the pressure of a fluid of hard spheres and in the solid phase we use the fit to simulation data of Hall [14]. In both cases we denote the hard-sphere free energy per sphere by $k_{B} T a_{\mathrm{HS}}$. For the free volume $\alpha$ we use the scaled particle theory expression $[4,15,16]$. The activity function is expressed as the product of a normalized distribution function $f_{z}$ and a total density $\rho_{r} / v_{0}\left(\rho_{r}\right.$ is the product of the density and a factor of $v_{0}$, which makes it dimensionless). We choose the Schultz distribution function $f_{z}(\varepsilon)[1,10]$; then

$$
z_{p}(\varepsilon)=\left(\rho_{r} / v_{0}\right) f_{z}(\varepsilon)
$$

where $v_{0}=(\pi / 6) \sigma^{3}$ is the volume of a colloidal sphere. This volume will be used to make our densities dimensionless. As $f_{z}(\varepsilon)$ is normalized, the integral of $z_{p}(\varepsilon)$ over $\varepsilon$ is equal to $\rho_{r} / v_{0}$. In the absence of a colloid the polymer is an ideal gas and the total polymer number density of a polydisperse polymer with $z_{p}(\varepsilon)$ given by Eq. (2) is $\rho_{r}$. The subscript $r$ of $\rho_{r}$ stands for reservoir; fixing the activity function of the polymer is equivalent to placing the system in contact with a reservoir of pure polymer with a density function given by the right-hand side of Eq. (2) [17]. The reservoir is separated from the system by a membrane that is permeable to a polymer but not to a colloid. The Schultz distribution function $f_{z}$ has two parameters [1]; $\varepsilon_{1}$ the first moment of $f_{z}$ and $z$, which determines the width of the distribution. The variance of $\varepsilon \operatorname{var}(\varepsilon)$ is given by

$$
\operatorname{var}(\varepsilon)=\frac{\varepsilon_{1}^{2}}{z+1} .
$$

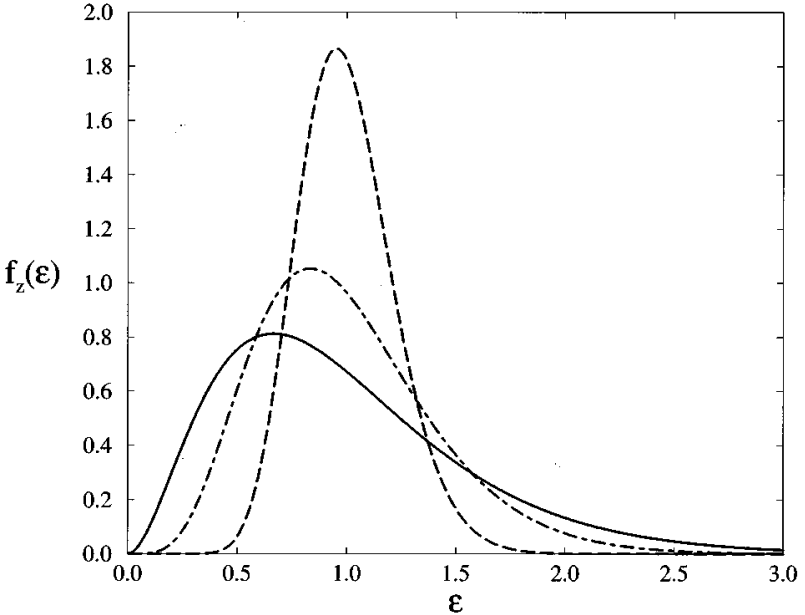

FIG. 2. Schultz distribution function $f_{z}$ with $\varepsilon_{1}=1$ and $z=2$ (solid curve), $z=5$ (dot-dashed curve), and $z=20$ (dashed curve).

In Sec. III we perform calculations for three values of $z: 2,5$, and 20. We show Schultz distributions with $\varepsilon_{1}=1$ for all three values of $z$ in Fig. 2. Finally, $\Omega$ is given by

$$
\frac{\beta \Omega\left(N_{c}, V, \rho_{r}, z, \varepsilon_{1}\right)}{N_{c}}=a_{\mathrm{HS}}(\eta)-\frac{\rho_{r}}{\eta} \int_{0}^{\infty} d(\varepsilon) f_{z}(\varepsilon) \alpha(\varepsilon, \eta),
$$

where the colloidal density is expressed in terms of its volume fraction $\eta=\left(N_{c} / V\right) v_{0}$. In the limit of $z \rightarrow \infty$ the Schultz distribution tends to a $\delta$ function centered on $\varepsilon=\varepsilon_{1}$ and Eq. (4) reduces to

$$
\frac{\beta \Omega\left(N_{c}, V, \rho_{r}, \varepsilon_{1}\right)}{N_{c}}=a_{\mathrm{HS}}(\eta)-\frac{\rho_{r}}{\eta} \alpha\left(\varepsilon_{1}, \eta\right) .
$$

Equation (5) is for a monodisperse polymer of size $\varepsilon_{1}$ [4]; $\rho_{r}$ is its activity and $\alpha\left(\varepsilon_{1}, \eta\right)$ the fraction of the volume available to a polymer coil. We now briefly discuss the approximations made in deriving Eqs. (4) and (5). There are two approximations; the first is decoupling the motion of the colloidal spheres and polymer coils so that the colloidal spheres move just as if the polymer was not there and the polymer coils move in the spaces left to them by the colloid $[4,9]$. With this approximation the change in semigrand potential on adding polymer, i.e., on increasing the polymer chemical potential from zero, are just equal to the total number of polymer coils in the system. The last terms of Eqs. (4) and (5) are just the ratios of the number of polymer coils to the number of colloidal particles. Thus the density function of the polymer $\rho_{p}(\varepsilon)$ is

$$
\rho_{p}(\varepsilon)=\left(\rho_{r} / v_{0}\right) f_{z}(\varepsilon) \alpha(\varepsilon, \eta)=\rho(\varepsilon) / v_{0},
$$

where the second equality merely defines a reduced polymer density function. The total reduced polymer density $\rho$ is just the integral of $\rho(\varepsilon)$ over $\varepsilon$. The second approximation consists of using approximate expressions for $a_{\mathrm{HS}}$ and $\alpha$. Although our $a_{\mathrm{HS}}$ is known to be very accurate, our expression for $\alpha$ was derived in the fluid phase $[15,16]$ and yet we also 
use it in the solid phase. See Ref. [8] for an evaluation of the accuracy of our expression for $\alpha$ in both the fluid and the solid phases.

For finite $z$ the integral in Eq. (4) is an effective $\alpha$ for the polymer; it is the average free volume for polymer coils distributed according to $f_{z}$. So we denote the integral of Eq. (4) by $\alpha_{\text {eff }}$; then

$$
\frac{\beta \Omega\left(N_{c}, V, \rho_{r}, z, \varepsilon_{1}\right)}{N_{c}}=a_{\mathrm{HS}}(\eta)-\frac{\rho_{r}}{\eta} \alpha_{\mathrm{eff}}\left(z, \varepsilon_{1}, \eta\right) .
$$

Whereas $\rho_{r}$ is the total number density in a pure polymer system in equilibrium with our polymer-colloid mixture, the total polymer density in the actual mixture is $\rho=\rho_{r} \alpha_{\text {eff }}$. The pressure $p$ and colloidal activity $\lambda$ may be derived from the semigrand potential (7); they are

$$
\beta p v_{0}=\beta p_{\mathrm{HS}} v_{0}+\rho_{r}\left[\alpha_{\mathrm{eff}}-\eta\left(\frac{\partial \alpha_{\mathrm{eff}}}{\partial \eta}\right)\right],
$$

where $p_{\mathrm{HS}}$ is the pressure of the fluid or solid of hard spheres, and

$$
\ln \lambda=\frac{\beta \Omega}{N_{c}}+\frac{\beta p v_{0}}{\eta} .
$$

\section{PHASE BEHAVIOR}

Our approximate theory for the colloid plus polydisperse polymer mixture yields a pressure (8) and chemical potential (9) that are analytical functions of the field variable $\rho_{r}$ and the density variable $\eta$ (for fixed $z$ and $\varepsilon_{1}$ ). (See Ref. [18] for a discussion of field and density variables.) This is completely analogous to a one-component system in the canonical ensemble, with $\rho_{r}$ taking the place of $T$. Thus our mixture, as described by Eq. (7), must obey the Gibbs phase rule for a one-component system. Although, in principle, an infinite number of phases can coexist in a polydisperse system, our approximate $\Omega$ will give coexistence between a maximum of three phases. Note that, for a fixed value of $z$, we have only two degrees of freedom $\rho_{r}$ and $\eta$ and so we would not expect to get more than three-phase coexistence from an exact $\Omega$.

With a monodisperse polymer Lekkerkerker et al. [4] found fluid-fluid coexistence for $\varepsilon_{1} \geqslant 0.32$. As this coexistence is the most characteristic feature of the colloid plus polymer mixtures it is of interest to see how it is affected by polydispersity of the polymer. We will compare a monodisperse mixture with polydisperse mixtures with $z=20,5$, and 2 . We choose the mixtures such that a colloidal sphere excludes the same volume on average to each of the four polymer systems, i.e., the polymer-colloid second virial coefficient is the same for all mixtures; see the Appendix. The first moments $\epsilon_{1}$ of the polymer mixtures are $0.4193,0.4136,0.4$, and 0.3829 for $z=\infty$ (monodisperse), 20, 5, and 2, respectively. Their second moments $\epsilon_{2}$, which are proportional to their average molecular weights, are $0.1738,0.1792,0.1867$, and 0.1955 .

Results are shown in Fig. 3 for monodisperse polymer with $\varepsilon_{1} \simeq 0.4193$ and three polymer mixtures with different polydispersities but the same polymer-colloid second virial

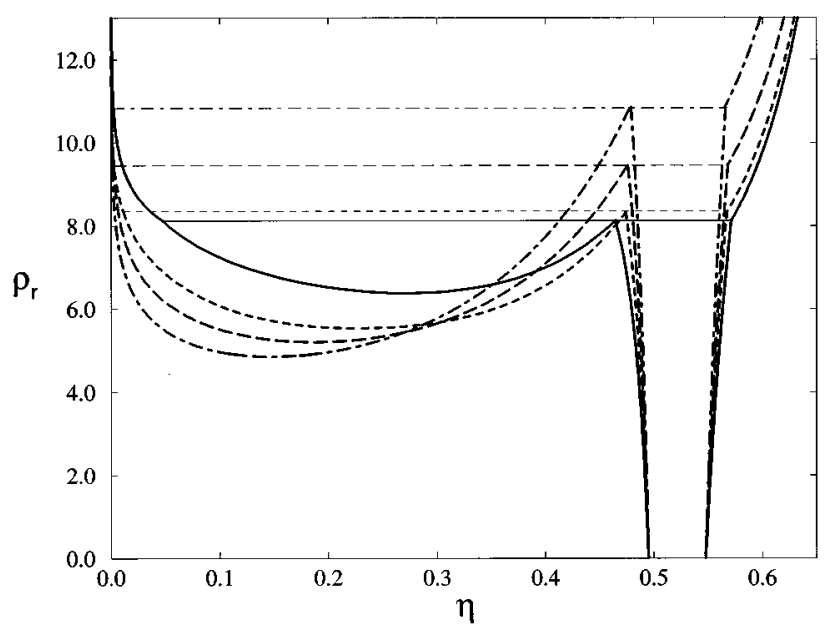

FIG. 3. Phase diagram in the $\rho_{r^{-}} \eta$ plane for the monodisperse polymer $\varepsilon_{1} \simeq 0.4193$ (solid curve) and polydisperse polymer $z=20, \varepsilon_{1} \simeq 0.4136$ (short-dashed curve), $z=5, \varepsilon_{1}=0.4$ (longdashed curve), and $z=2, \varepsilon_{1} \simeq 0.3829$ (dot-dashed curve). The thin horizontal lines connect the three coexisting densities at the triple points.

coefficient. Clearly, increasing the polydispersity increases the extent of the fluid-fluid coexistence. This is also apparent in the $\rho-\eta$ plane; see Fig. 4 . In Fig. 3 we see that as the polydispersity increases the polymer activity $\rho_{r}$ at the critical point decreases, while that at the triple point increases. The range of $\rho_{r}$ over which there is coexistence between colloidpoor and colloid-rich fluid phases is only $\simeq 1.75$ for monodisperse polymer, but for $z=2$ it is $\simeq 5.97$. In addition, as the polydispersity increases the volume fraction of the colloid at the demixing critical point decreases. In Fig. 4 the total area enclosed by the fluid-fluid coexistence curve and an edge of the three-phase triangle is considerably larger for the polydisperse polymer than for the monodisperse one.

Also worthy of note is the much larger total polymer density of the polydisperse polymer at the two colloid-rich corners of the three-phase triangle in Fig. 4. In order to see why

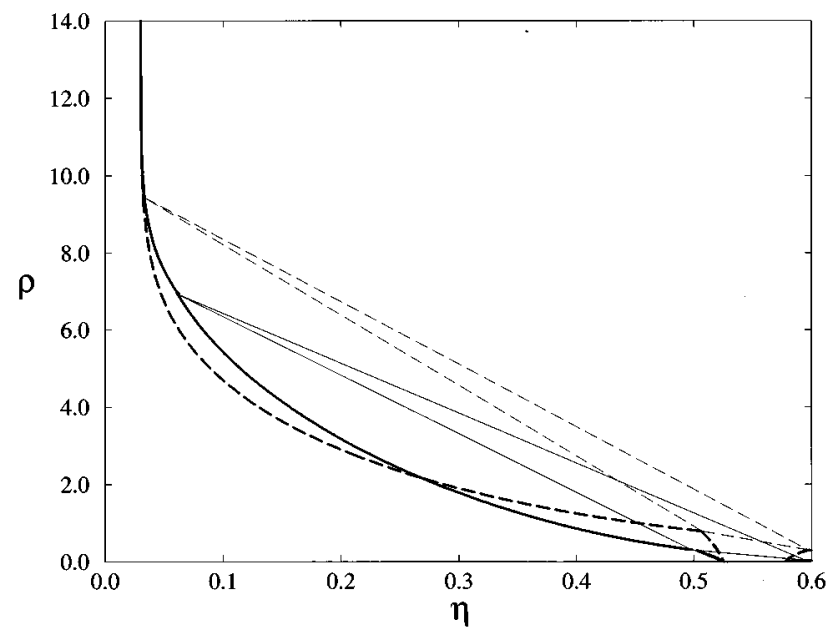

FIG. 4. Phase diagram in the $\rho-\eta$ plane for monodisperse polymer $\varepsilon_{1} \simeq 0.4193$ (solid curve) and polydisperse polymer $z=5$, $\varepsilon_{1}=0.4$ (dashed curve). The thin lines are the edges of the three phase triangles. 


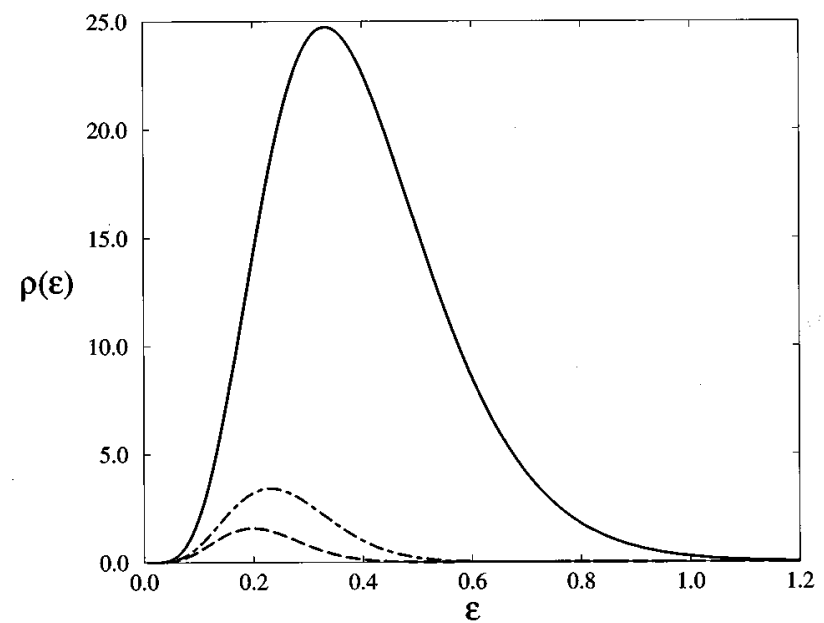

FIG. 5. Density function $\rho(\varepsilon)$ for the three coexisting phases at the triple point for $\varepsilon=0.4$ and $z=5$. The solid curve is for the lowest colloid volume fraction $\eta$ and the dashed curve is for the highest $\eta$.

this is so we examine the full density function of the polymer $\rho_{p}(\varepsilon)$; it is is plotted in Fig. 5 for the three coexisting phases at the triple point of the mixture with $\varepsilon=0.4$ and $z=5$. Of course the polymer density is much higher in the colloidpoor phase, but note that the position of the maximum in $\rho(\varepsilon)$ shifts to smaller values of $\varepsilon$ at larger colloid volume fractions. In the colloid-poor phase (solid curve in Fig. 5) the polymer density is highest near $\varepsilon \approx 0.4$, but in the highest$\eta$ phase (dashed curve) there is very little polymer for these values of $\varepsilon$; most of the polymer is near $\varepsilon \approx 0.2$. This is due to the fact that at high $\eta, \alpha$ is a rapidly decreasing function of $\varepsilon$; there is very little free volume available to polymer coils of size $\varepsilon \approx 0.4$ at high $\eta$, but much more for coils with $\varepsilon \approx 0.2$. The very small value of $\alpha$ for $\varepsilon=0.4$ is also the reason why the polymer density is so low in the colloid rich phases at the triple point of the monodisperse polymer. We conclude that the polymer density is much higher at higher colloid volume fractions if the polymer is polydisperse because the polydisperse polymer contains some coils considerably smaller than $\varepsilon_{1}$ that have much more free volume at high colloidal densities than do coils with $\varepsilon=\varepsilon_{1}$.

There are (at least) two ways of thinking about the increase in fluid-fluid coexistence found for polydisperse polymer compared with that for monodisperse. The first is to recall that on going from a pure system to a mixture the density jump at first-order transitions almost always increases. This is due to partitioning of the components of the mixture between the two coexisting phases. Here partitioning of the different polymer sizes actually encourages the formation of the first-order phase separation. Polydispersity should quite generally encourage first-order transitions as the presence of two coexisting phases allows the polydisperse component to distribute itself between the two phases in such a way as to minimize the semigrand potential or free energy. Second, in Fig. 5, we can see that the large- $\varepsilon$ tails of the polymer distributions are almost completely excluded from the colloid-rich phases. As $\eta$ increases, $\alpha$ at these large values of $\varepsilon$ decreases very rapidly, so creating the condition for coexistence between a colloid-poor, polymer-rich and a colloid-rich, polymer-poor phase. On increasing the polydis-

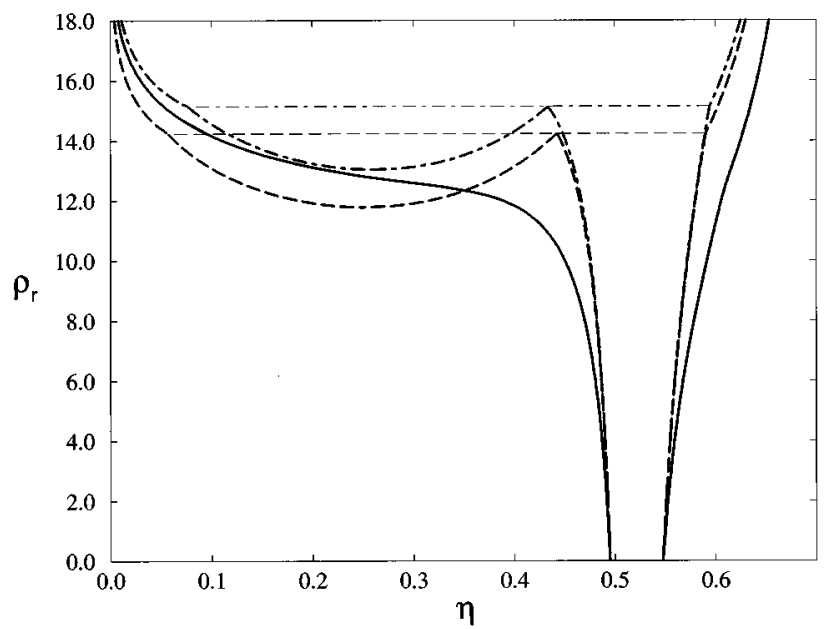

FIG. 6. Phase diagram in the $\rho_{r}-\eta$ plane for the monodisperse polymer $\varepsilon_{1}=0.3$ (solid curve) and polydisperse polymer $z=5$, $\varepsilon_{1} \simeq 0.2890$ (dashed curve) and $z=5, \varepsilon_{1} \simeq 0.2777$ (dot-dashed curve). The thin horizontal lines connect the three coexisting densities at the triple points. The first polydisperse polymer has the same $B_{p}$ as the monodisperse polymer. The second polydisperse polymer (dot-dashed curve) has $\epsilon_{2}=0.09$, the same second moment of $\epsilon$, and hence the same average molecular weight, as the monodisperse polymer.

persity we increase the number of large and small polymer coils at the expense of polymer coils of average size. It is the introduction of these large polymer coils that is the predominant way in which polydispersity increases the driving force towards phase separation.

Finally, if polydispersity encourages fluid-fluid coexistence then we should be able to find coexistence for smaller (average) polymer sizes than for monodisperse polymer. In Fig. 6 we show that, indeed, we can find coexistence even when a monodisperse polymer with the same polymercolloid second virial coefficient or the same average molecular weight is too small to induce fluid-fluid coexistence.

\section{CONCLUSION}

Polydispersity has been found to increase the extent of fluid-fluid coexistence. Thus, if a polymer is added to a colloidal suspension in order to induce phase separation it would be advantageous to use a polydisperse, not monodisperse, polymer. Although this finding is not particularly surprising, it does go against the prevailing tendency to try to minimize the polydispersity of polymer. Traditionally, the inevitable polydispersity of polymers is regarded as an irritation. In the sense that polydispersity complicates the theoretical study and understanding of polymeric systems it is reasonable to regard it as a problem. However, from the point of view of using the polymer to produce a specific effect polydispersity is, a priori, as likely to help as to hinder.

The partitioning of the polymer between the colloid-rich and colloid-poor phases is similar to that found in a polymer solution in contact with a porous medium [19]. In the porous medium the ratio of the density of small coils to the density of large polymer coils is larger than in the pure polymer. Here we found that this ratio is larger in the colloid-rich 
phase than in the colloid-poor phase. Thus a colloidal suspension could be used to selectively extract part of the polymer distribution. From Fig. 5 we see that the colloid-rich phases have predominantly small polymer coils and so if we separate the coexisting phases and remove the colloid we are left with two polymer solutions. The solution that was colloid-rich now contains polymer coils with an average size $\varepsilon_{1}$ about half that we started with.

\section{ACKNOWLEDGMENTS}

It is a pleasure to acknowledge a careful reading of the manuscript by I. Pagonabarraga. R.P.S. would like to thank The Royal Society for financial support and the FOM institute AMOLF for its hospitality. The work of the FOM Institute is part of the research program of FOM and is made possible by financial support from the Netherlands Organisation for Scientific Research (NWO).

\section{APPENDIX}

The second virial coefficient $B_{2}^{m}$ for the interaction between a colloidal sphere and a polymer coil of size $\varepsilon$ is

$$
B_{2}^{m}=(\pi / 6)(1+\varepsilon)^{3} \sigma^{3} .
$$

Using the Asakura-Oosawa [6,7] model of the colloidpolymer interaction a colloidal sphere interacts with the polymer coil as if the coil was a hard sphere of diameter $\varepsilon \sigma$. The second virial coefficient is then the volume excluded by a hard-sphere of diameter $\sigma$ to one of diameter $\varepsilon \sigma$. If the polymer is monodisperse then Eq. (A1) gives the second viral coefficient straightaway. However, if the polymer is polydisperse we must average the excluded volume over the distribution of polymer sizes

$$
\begin{aligned}
B_{2}^{p} & =\left(\frac{\pi}{6}\right) \sigma^{3} \int d(\varepsilon) f_{z}(\varepsilon)(1+\varepsilon)^{3} \\
& =\left(\frac{\pi}{6}\right) \sigma^{3}\left(1+3 \varepsilon_{1}+3 \varepsilon_{2}+\varepsilon_{3}\right),
\end{aligned}
$$

where $\varepsilon_{i}$ is the $i$ th moment of $f_{z}(\varepsilon)[1]$ and $B_{2}^{p}$ is the second virial coefficient for polydisperse polymer, i.e., the coefficient of $\rho \eta / v_{0}^{2}$ in the density expansion for the pressure. For the Schultz distribution we can express all moments of $f_{z}(\varepsilon)$ in terms of $z$ and $\varepsilon_{1}$, then

$$
B_{2}^{p}=\left(\frac{\pi}{6}\right) \sigma^{3}\left(1+3 \varepsilon_{1}+\frac{3(z+2)}{z+1} \varepsilon_{1}^{2}+\frac{(z+3)(z+2)}{(z+1)^{2}} \varepsilon_{1}^{3}\right) .
$$

When comparing mixtures of different polydispersities and poly- and monodisperse mixtures we keep $B_{2}^{p}$ the same for all mixtures and equal to $B_{2}^{m}$ for the monodisperse polymer. This is easily achieved as given $B_{2}^{p}$ we can choose a value for $z$ and then solve Eq. (A3) for $\varepsilon_{1}$. The value of $\varepsilon_{1}$ thus obtained decreases with increasing $z$.

We are assuming that the polymer coils are ideal and that the size of a coil $\varepsilon$ is proportional to its radius of gyration $[8,9]$. The radius of gyration of ideal polymer coils scales as $N_{m}^{1 / 2}$ where $N_{m}$ is the number of monomers of the coil. So, $N_{m} \sim \varepsilon^{2}$ and the average number of monomers of a polymer in our polydisperse polymer mixtures is proportional to $\varepsilon_{2}$. Increasing the polydispersity at constant $B_{2}^{p}$ causes $\varepsilon_{2}$ to increase. Therefore, the polydisperse mixtures of Fig. 3 have a higher average $N_{m}$ and therefore a higher average molecular weight than the the monodisperse polymer.
[1] J. J. Salacuse and G. Stell, J. Chem. Phys. 77, 3714 (1982).

[2] W. C. K. Poon and P. N. Pusey, in Observation, Prediction and Simulation of Phase Transitions in Complex Fluids, edited by M. Baus et al. (Kluwer, Dordrecht, 1995).

[3] D. Rosenbaum, P. C. Zamora, and C. F. Zukoski, Phys. Rev. Lett. 76, 150 (1996).

[4] H. N. W. Lekkerkerker, W. C.-K. Poon, P. N. Pusey, A. Stroobants, and P. B. Warren, Europhys. Lett. 20, 559 (1992).

[5] N. A. M. Verhaegh, J. S. van Duijneveldt, and H. N. W. Lekkerkerker, J. Chem. Phys. 102, 1416 (1995).

[6] S. Asakura and F. Oosawa, J. Chem. Phys. 22, 1255 (1954).

[7] A. Vrij, Pure Appl. Chem. 48, 471 (1976).

[8] E. J. Meijer and D. Frenkel, J. Chem. Phys. 100, 6873 (1994).

[9] P. Warren, S. M. Ilett, and W. C. K. Poon, Phys. Rev. E 52, 5205 (1995).

[10] J. A. Gualtieri, J. M. Kincaid, and G. Morrison, J. Chem. Phys.
77, 521 (1982).

[11] J. G. Briano and E. D. Glandt, J. Chem. Phys. 80, 3336 (1984).

[12] R. P. Sear and G. Jackson, J. Chem. Phys. 103, 8684 (1995).

[13] N. E. Carnahan and K. E. Starling, J. Chem. Phys. 51, 635 (1969).

[14] K. R. Hall, J. Chem. Phys. 57, 2252 (1972).

[15] H. Reiss, H. L. Frisch, and J. L. Lebowitz, J. Chem. Phys. 31, 369 (1959).

[16] H. Reiss, J. Phys. Chem. 96, 4736 (1992).

[17] H. N. W. Lekkerkerker, P. Buining, J. Buitenhuis, G. J. Vroege, and A. Stroobants, in Observation, Prediction and Simulation of Phase Transitions in Complex Fluids (Ref. [2]).

[18] J. S. Rowlinson and F. L. Swinton, Liquids and Liquid Mixtures (Butterworths, London, 1982).

[19] I. Teraoka, Z. Zhou, K. H. Langley, and F. E. Karosz, Macromolecules 26, 3223 (1993). 